Devel opment of DSP Unit for Onl i ne Tuning and Appl i cat i on to Neural Pat ter $n$ Recogniti on Syst em

\begin{tabular}{|l|l|}
\hline 著者 & Sat oh H r onobu, Takeda Fumi aki \\
\hline $\begin{array}{l}\text { j our nal or } \\
\text { publ i cat i on t i t l e }\end{array}$ & Lect ure Not es i n Comput er Sci ence \\
\hline vol une & 3213 \\
\hline page range & $866-873$ \\
\hline year & 2004 10-14 \\
\hline URL & ht t p: //hdl . handl e. net /10173/613 \\
\hline
\end{tabular}




\title{
Development of DSP unit for Online Tuning and Application to Neural Pattern Recognition System
}

\author{
Hironobu Satoh and Fumiaki Takeda
}

Development of Intelligent Mechanical System Engineering, Kochi University of Technology 185 Miyanokuchi, Tosayamada-cho, Kami-Gun, Kochi 782-8502, Japan 075041b@gs.kochi-tech.ac.jp

\begin{abstract}
Recently, smaller and more effective recognition system is required in various fields. In our previous researches, the recognition system using neural network $(\mathrm{NN})$ and DSP had been developed. In some research fields, such as biometrics, the target of the system was to recognize patterns whose data varied widely because of the difference of individuals and surroundings, but its recognition ability was not enough. So the online tuning system is proposed in this paper. The proposed system, which is consisted of DSP unit and the NN continuous learning and recognition parts, is used to recognize the patterns whose data varied widely. We discuss to adjust the recognition system to data varied widely by continuous leaning. In experiment, performance of the online tuning system is checked, and we attempt to apply the system to electromyogram (EMG) pattern recognition.
\end{abstract}

\section{Introduction}

Recently, smaller and effective recognition system was needed in various fields. In our previous researches, some flexible recognition systems using the neural network (NN) were developed, and to make the system smaller, more portable and more efficient, the digital signal processor (DSP) was applied to its operation units. However, the system aimed at some targets whose data variation caused by the difference of individuals and surroundings was widely. Its recognition ability is not enough. The reason was that, initial learning data is generated for include a target for recognition. Then, initial learning data is used for first learning by the recognition system. So, the NN can not adjust data varied widely because of the process. To solve this problem, we develop the system that can input the learning data online, and learn continuously described online tuning system. Because of continuous learning can respectively extract the characteristics of each recognition targets, it is possible that the system recognize the target whose data varied, widely.

In this paper, we discuss the problem that the system's recognition ability for some cases whose data varied widely is not satisfied, and proposed a solution of using the online tuning system, which is realized on the DSP unit. In the experiment, a voltage controlled oscillator (VCO) is used to simulate the EMG data, which is a minute electricity. After that, it is used as learning data to verity the DSP unit of the online tuning system. Finally, the real electromyogram $(E M G)$ pattern is used to examine the online tuning system. 


\section{Online tuning system}

In this section, the problems of the previous recognition system are discussed, and to solve the problems, the online tuning system is proposed.

\section{2-1 Previous the NN recognition system}

The previous NN recognition system has been used in banknotes recognition system and EMG patterns recognition system [1],[2],[3]. Previous research, recognition ability was not satisfied with the case whose data varied so large. Individual variation and differences of the environment influenced sample data[4],[5],[6]. General learning data are sampled by the recognition system. Then the recognition system starts learning. And the initial learning is converged using sampled learning data. But the data varied widely is difficult to converge in the process of the learning. Because, the learning data is increased to increase generalization ability. In addition, sampling and editing learning data is difficult to include all learning data. So, the imperfect learning data was used in initial learning and the recognition ability of the NN is not enough for unknown data. In the result, recognition ability of the proposed recognition system which is trained by imperfect learning data is low.

Proposed recognition system is applied in various fields of the system, EMG recognition system is one of the examples. EMG data for learning and recognition data is influenced by differences of individuals and the environment. So, recognition ability is low.

\section{2-2 Online tuning system}

To solve the problems, the system, which can obtain the additional learning data and complete the continuous learning, is proposed. Fig.1. shows the process of the online tuning system. First, initial NN weight is on generated the personal computer (PC). The initial NN weight has ability which is able to recognize a wide use. Then, the weight is transported to the DSP unit. After that the continuous learning is completed on the DSP unit. Finally, after the system converged, the final weight is generated for special individual data. Because of the process of continuous learning using the additional data, the recognition learning ability of the system is improved, even if the data of recognized targets varied, widely.

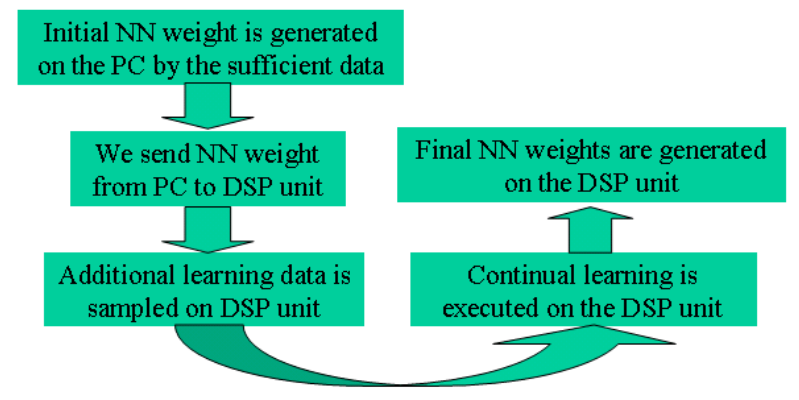

Fig.1. Process of the online tuning system 


\section{DSP unit for online tuning}

Table 1. Comparison between previous DSP unit and online tuning DSP unit

\begin{tabular}{|l|l|l|}
\hline & $\begin{array}{l}\text { Previous DSP } \\
\text { unit }\end{array}$ & $\begin{array}{l}\text { Online tuning DSP } \\
\text { unit }\end{array}$ \\
\hline Input data & Digital data & Analog data \\
\hline A/D converter & None (on PC) & Possess (on unit) \\
\hline FFT & None (on PC) & Possess (on unit) \\
\hline Rotating slab value for learning & None (on PC) & Possess (on unit) \\
\hline
\end{tabular}

Then we develop new DSP unit for online tuning. Table.1 shows the differences between the previous DSP unit and proposed online tuning DSP unit. Moreover, the pre-processing for learning and learning are executed on the DSP unit, respectively.

\section{3-1 Structures of the hardware}

Because of the characteristics of DSP, such as ALU, multiplier they are suitable for the NN computation. It is simple for programmers to develop software. The initialization data is saved in EPROM, and the program of the NN learning and recognition are saved in flash memory. Once the DSP unit start, the program is send to S-RAM. The alternation of learning and recognition mode is controlled by the mode switch. The serial port is used to communicate with PC. LED and LCD are used to display the state of the DSP unit. Fig.2. shows construction of proposed DSP unit. Fig.3. shows the outside and inside.

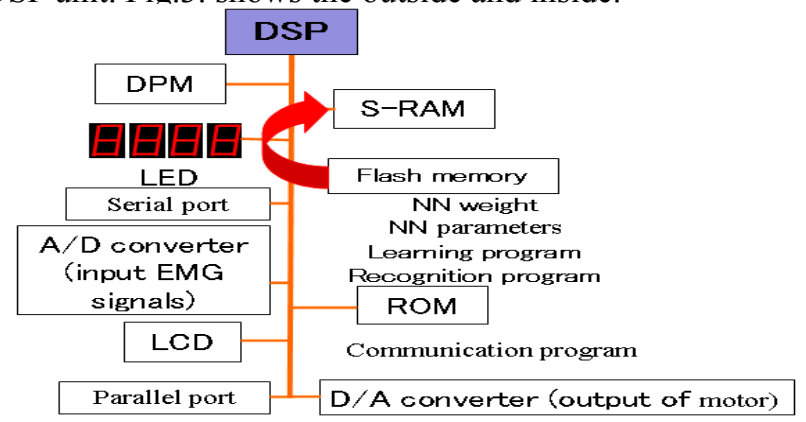

Fig.2. Construction of proposed DSP unit

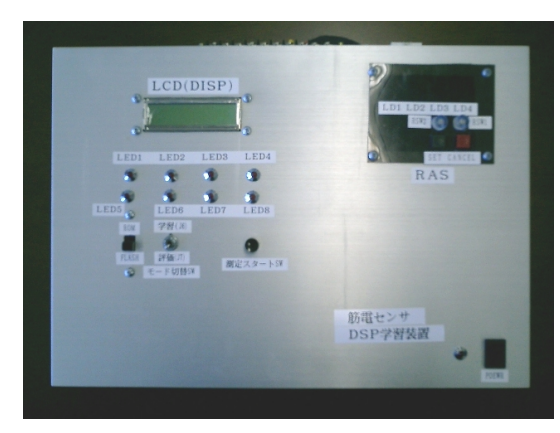

(a) The outside of DSP unit

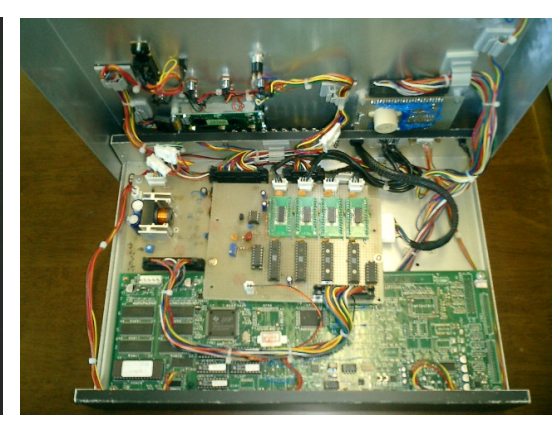

(b) The inside of DSP unit

Fig.3. Feature of DSP unit 
On the proposed DSP unit for online tuning system, there are 4 analog input and 8 analog output terminals whose resolution is 8 bit. Still more, it is possible that the DSP unit completed the learning and recognizing process, independently.

\section{3-2 Structures of the software}

The back propagation (BP) algorithm is applied in the NN learning, and the program is written in C language. Fig.4. illustrates the flow chart of the DSP software. As starting of the DSP unit, the learning and recognizing program are send from flash memory to S-RAM, as mentioned on section 3-1. Since the acquirement of learning data should be executed by DSP unit, independently, the function of online inputting of the analog data is expanded. After the data input to the DSP unit, it is transformed using the FFT algorithm. After that it is converted to input data for the $\mathrm{NN}$ and is applied on learning and recognition process.

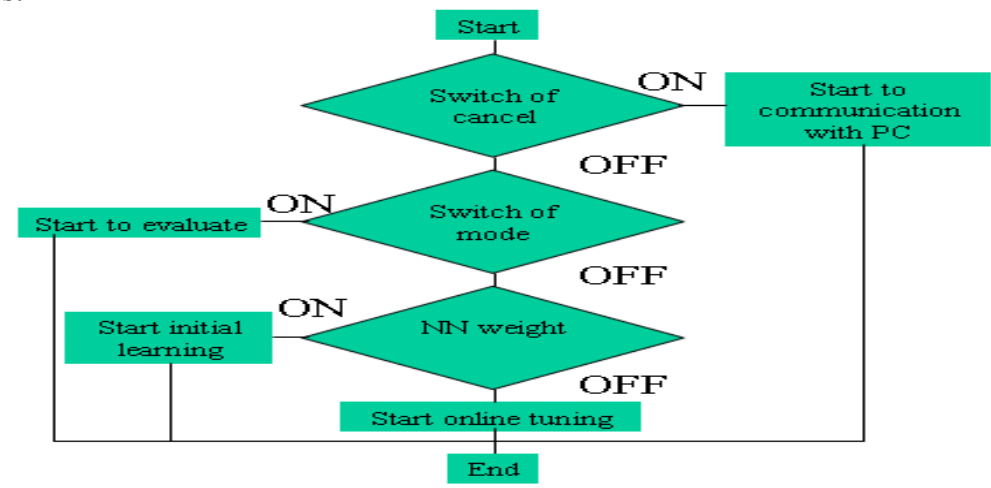

Fig.4. Flow chart of the DSP software

\section{Verification of DSP unit}

In this section, the online tuning function of the DSP unit is verified. The intention of this verification is that the system can be applied on EMG pattern recognition. First, the sin wave signal generated by voltage controlled oscillator (VCO) is used to simulate the EMG signal because of its feebleness and then the sine signal is used to verity the proposed system. After that, the EMG signals of 7 arm actions including idling, right click, left click, up, down, right, left are used to verify the online tuning system.

\section{4-1 Condition of the experiment}

In the experiment, 7 kind of sine wave signal with $1 \mathrm{KHz}$ is generated by VCO. They are inputted to the DSP unit for learning and recognition processes. First, the NN weight is initialized on PC by the initial learning. Then the sine wave signals generated by VCO are inputted to DSP unit from the analog input terminals. After that, the signal is converted using A/D converter and FFT algorithm to get frequency signal. Finally, the frequency signal is transformed to 
the input for the NN being used to learning and recognizing processes. Fig.5. shows the different slab values of 7 sine wave signals. Table2 shows the condition of the NN learning. Fig.6. shows VCO.

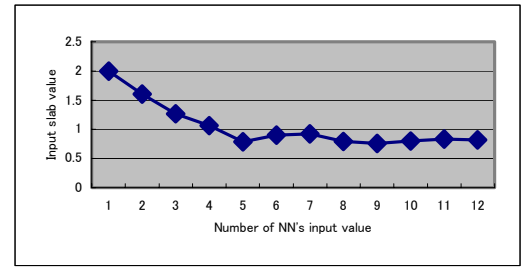

(a) input 1

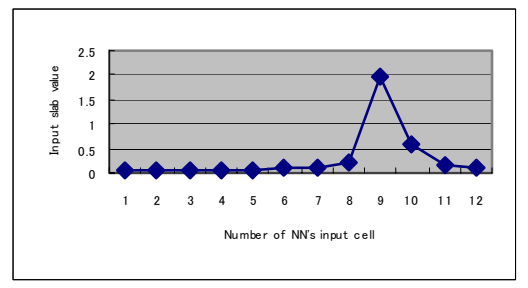

(c) input 3

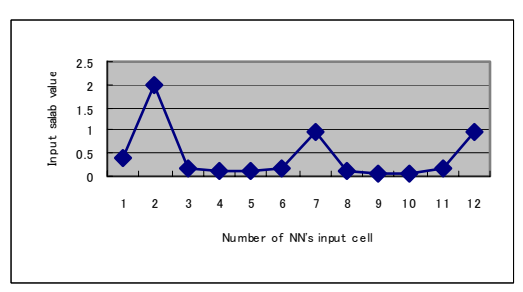

(e) input 5

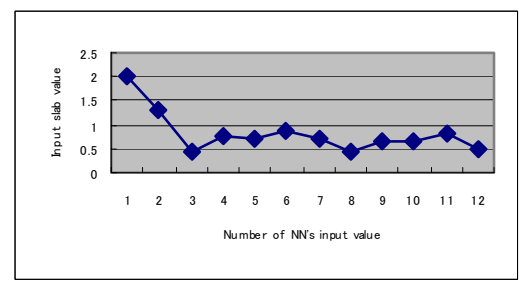

(g) input 7

Fig.5. The different slab value of 7 sine wave

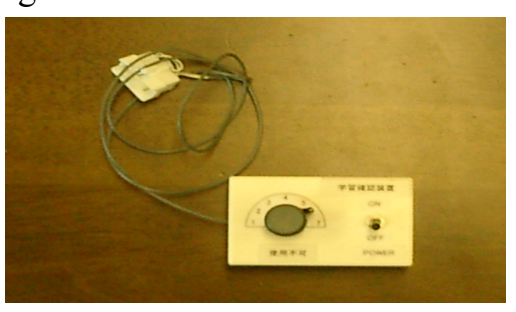

Fig.6. VCO

(d) input 4

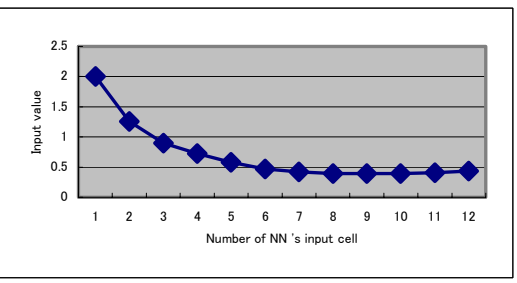

(b) input 2
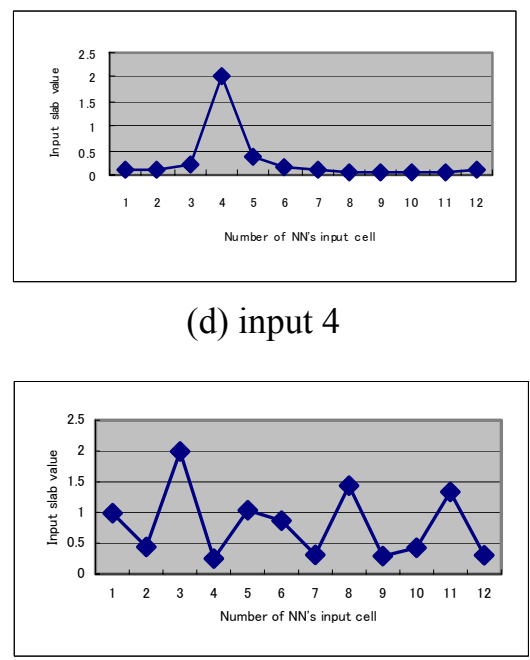

(f) input 6 
Table 2. Experimental conditions

\begin{tabular}{|l|l|}
\hline Mean squared error & 0.0001 \\
\hline Maximum iteration number & 20000 \\
\hline Learning method & Improved BP method (eq. 1 ) \\
\hline Construction of the NN & $48 \times 25 \times 7$ \\
\hline Number of learning data for a sampling & 3 \\
\hline Learning constant $\eta$ & 0.05 \\
\hline Inertial constant $\alpha$ & 0.95 \\
\hline Vibrations constant $\beta$ & -0.1 \\
\hline
\end{tabular}

$$
\Delta W_{i}(t)=-7 \delta_{k} O_{k}+\alpha \Delta W_{k}(t-1)+\beta \Delta W_{i g}(t-2)
$$

\section{4-2 Result of verification}

The results of the experiment are showed in table3. Fig.7. shows process of examination.

Table 3. Experimental result with $\mathrm{VCO}$

\begin{tabular}{|l|c|}
\hline Pattern & Recognition rate (\%) per 20 \\
\hline 1 & $100 \%$ \\
\hline 2 & $100 \%$ \\
\hline 3 & $100 \%$ \\
\hline 4 & $100 \%$ \\
\hline 5 & $100 \%$ \\
\hline 6 & $100 \%$ \\
\hline 7 & $100 \%$ \\
\hline
\end{tabular}

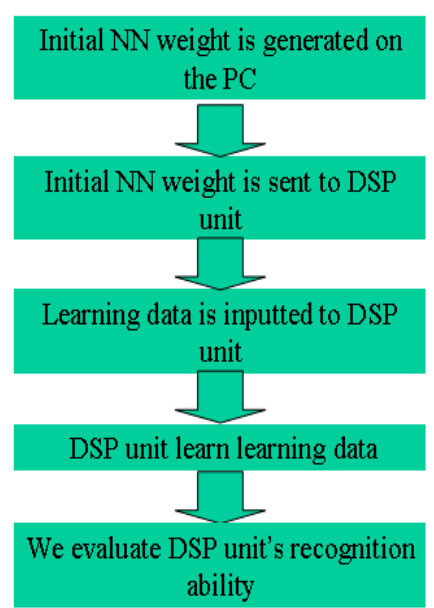

Fig.7. Process of exmination

\section{4-3 Examination}

In results of the verification, the DSP unit for online tuning was effective when the $4 \mathrm{ch}$ analog signals were sampled and used in learning and recognition on the DSP unit. Moreover, the we checked the performance of DSP unit for online tuning is verified using EMG real data. 


\section{4-4 Experiment using EMG real data}

Table 4. Results of experimentation

\begin{tabular}{|c|c|c|c|}
\hline & Testee A & Testee B & Testee C \\
\hline 5 samples & $51.43 \%$ & $47.14 \%$ & $26.43 \%$ \\
\hline 10 samples & $73.57 \%$ & $63.57 \%$ & $35.00 \%$ \\
\hline
\end{tabular}

In this section, the online tuning system is verified using the EMG real data, and the effectiveness of continuous learning is shown. The EMG is easily influenced by the differences of individuals and changes of environment. So the difference of individuals could not be recognized correctly only after the initial leaning.

In the experiment, the 7 patterns of arm motion (Extension, Flexion, Pronation, Supination, Open, Grasp, Idking) is used as learning samples. Each of them is sampled 5 and 10 times respectively. The respective motion is evaluated 20 times. Table 4 shows the results of experiment using he online tuning system for 3 examinees. From the table we can see that the recognition ratio is improved while increasing the learning samples, and while the continuous learning is executed.

\section{Conclusion}

In this paper, we indicated problems of the previous system using the $\mathrm{NN}$ and defined. Still more, we indicated that the tuning means learning data is sampled online and the continuous learning is done using the sampled data. We designed the DSP unit for online tuning and verified them. This examination was considered to be applied to the EMG pattern recognition system using the new DSP unit. We used analog signals which were generated by VCO in examination. Results of the examination showed the system recognized the patterns with $100 \%$. Moreover, we checked performance of DSP unit for online tuning. Online tuning system was expanded to EMG pattern recognition system. Seven type of pattern (Extension, Flexion, Pronation, Supination, Opnen, Grasp, Idling) were used to verify the EMG recognition system using the online tuning system. Results of examination show recognition ability improved when the learning data were increased. We consider the online tuning system was effective on EMG pattern recognition system. In the future, we will try again to examine EMG pattern recognition system using online tuning system in detail.

\section{Reference}

1. F.Takeda, M.Nakahara, K.Nakaura and Y.Yamamoto.:Recognition System of Arm Behavior with Layered Neural Network, KES2001, pp.1003-1008, 2001

2. M.Nakahara, F.Takeda, K.Nakaura and Y.Yamamoto.: Propose of an Arm Behavior Recognition System with EMG and Neural Network:, ICMIT'01, pp.400-405, 2001

3. H.Satoh, F.Takeda.:Development of a Intelligent input Interface using EMG for MobileTerminal': , ISAGA, chiba, pp.1078-1088, 2003

4. F.Takeda, S.Omatu, Y.Matumoto.:Development of High Speed Neuro-Recgnition Unit and Application for Paper Curency, The International Workshop on Signal Processing Application and Tecnhology,pp.49-56

5. F.Takeada, M.Nakahara, Y.Ichiryu, H.Uchida.:Autonomic Neuro-Recognition Board for Paper Currecny:,APAT2000,pp.85-90,2000

6. F.Takeda, S.Omatu.:Neural Network Systems Technique and Applications in Paper Currency Recognition:, Neural Network Syst., Techniq. and Appli. ACADEMIC Press5, Chapter 4, p133-160,1998 